\title{
Time Series Data Augmentation for Deep Learning: A Survey
}

\author{
Qingsong Wen ${ }^{1}$, Liang Sun ${ }^{1}$, Fan Yang ${ }^{2}$, Xiaomin Song ${ }^{1}$, Jingkun Gao ${ }^{3 *}$, Xue Wang ${ }^{1}$, Huan Xu ${ }^{2}$ \\ ${ }^{1}$ DAMO Academy, Alibaba Group, Bellevue, WA, USA \\ ${ }^{2}$ Alibaba Group, Hangzhou, China \\ ${ }^{3}$ Twitter, Seattle, WA, USA \\ \{qingsong.wen, liang.sun, fanyang.yf, xiaomin.song, xue.w, huan.xu\}@alibaba-inc.com, \\ jingkung@twitter.com
}

\begin{abstract}
Deep learning performs remarkably well on many time series analysis tasks recently. The superior performance of deep neural networks relies heavily on a large number of training data to avoid overfitting. However, the labeled data of many realworld time series applications may be limited such as classification in medical time series and anomaly detection in AIOps. As an effective way to enhance the size and quality of the training data, data augmentation is crucial to the successful application of deep learning models on time series data. In this paper, we systematically review different data augmentation methods for time series. We propose a taxonomy for the reviewed methods, and then provide a structured review for these methods by highlighting their strengths and limitations. We also empirically compare different data augmentation methods for different tasks including time series classification, anomaly detection, and forecasting. Finally, we discuss and highlight five future directions to provide useful research guidance.
\end{abstract}

\section{Introduction}

Deep learning has achieved remarkable success in many fields, including computer vision (CV), natural language processing (NLP), and speech processing, etc. Recently, it is increasingly embraced for solving time series related tasks, including time series classification [Fawaz et al., 2019], time series forecasting [Han et al., 2019], and time series anomaly detection [Gamboa, 2017]. The success of deep learning relies heavily on a large number of training data to avoid overfitting. Unfortunately, many time series tasks do not have enough labeled data. As an effective tool to enhance the size and quality of the training data, data augmentation is crucial to the successful application of deep learning models. The basic idea of data augmentation is to generate synthetic dataset covering unexplored input space while maintaining correct labels. Data augmentation has shown its effectiveness in many applications, such as AlexNet [Krizhevsky et al., 2012] for ImageNet classification.

\footnotetext{
${ }^{*}$ The work was done when Jingkun Gao was at Alibaba Group.
}

However, less attention has been paid to find better data augmentation methods specifically for time series data. Here we highlight some challenges arising from data augmentation methods for time series data. Firstly, the intrinsic properties of time series data are not fully utilized in current data augmentation methods. One unique property of time series data is the so-called temporal dependency. Unlike image data, the time series data can be transformed into the frequency and time-frequency domains and effective data augmentation methods can be designed and implemented in the transformed domain. This becomes more complicated when we model multivariate time series where we need to consider the potentially complex dynamics of these variables across time. Thus, simply applying those data augmentation methods from image and speech processing may not result in valid synthetic data. Secondly, the data augmentation methods are also task dependent. For example, the data augmentation methods applicable for time series classification may not be valid for time series anomaly detection. In addition, data augmentation becomes more crucial in many time series classification problems where class imbalance is often observed. In this case, how to effective generate a large number of synthetic data with labels with less samples remains a challenge.

Unlike data augmentation for CV [Shorten and Khoshgoftaar, 2019] or speech [Cui et al., 2015], data augmentation for time series has not yet been comprehensively and systematically reviewed to the best of our knowledge. One work closely related to ours is [Iwana and Uchida, 2020] which presents a survey of existing data augmentation methods for time series classification. However, it does not review the data augmentation methods for other common tasks like time series forecasting [Bandara et al., 2020; Hu et al., 2020; Lee and Kim, 2020] and anomaly detection [Lim et al., 2018; Zhou et al., 2019; Gao et al., 2020]. Furthermore, the potential avenues for future research opportunities of time series data augmentations are also missing.

In this paper, we aim to fill the aforementioned gaps by summarizing existing time series data augmentation methods in common tasks, including time series forecasting, anomaly detection, classification, as well as providing insightful future directions. To this end, we propose a taxonomy of data augmentation methods for time series, as illustrated in Fig. 1. Based on the taxonomy, we review these data augmentation methods systematically. We start the discussion from the sim- 


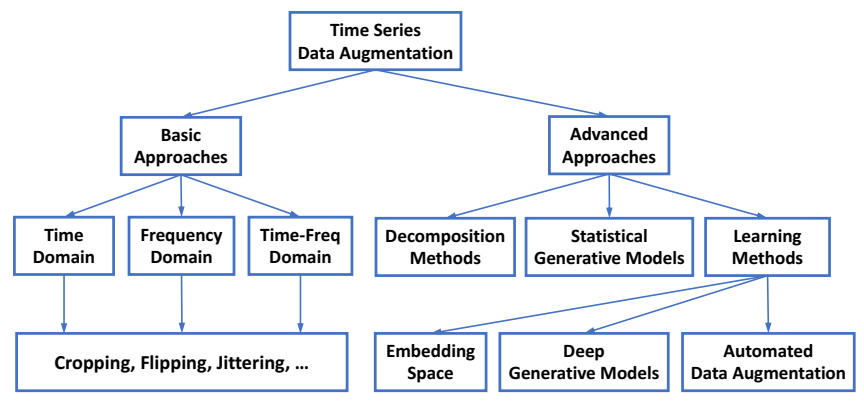

Figure 1: A taxonomy of time series data augmentation techniques.

ple transformations in time domain first. And then we discuss more transformations on time series in the transformed frequency and time-frequency domains. Besides the transformations in different domains for time series, we also summarize more advanced methods, including decomposition-based methods, model-based methods, and learning-based methods. For learning-based methods, we further divide them into embedding space, deep generative models (DGMs), and automated data augmentation methods. To demonstrate effectiveness of data augmentation, we conduct preliminary evaluation of augmentation methods in three typical time series tasks, including time series classification, anomaly detection, and forecasting. Finally, we discuss and highlight five future directions: augmentation in time-frequency domain, augmentation for imbalanced class, augmentation selection and combination, augmentation with Gaussian processes, and augmentation with deep generative models.

\section{Basic Data Augmentation Methods 2.1 Time Domain}

The transforms in the time domain are the most straightforward data augmentation methods for time series data. Most of them manipulate the original input time series directly, like injecting Gaussian noise or more complicated noise patterns such as spike, step-like trend, and slope-like trend. Besides this straightforward methods, we will also discuss a particular data augmentation method for time series anomaly detection, i.e., label expansion in the time domain.

Window cropping or slicing has been mentioned in [Le Guennec et al., 2016]. Introduced in [Cui et al., 2016], window cropping is similar to cropping in CV area. It is a subsample method to randomly extract continuous slices from the original time series. The length of the slice is a tunable parameter. For classification problem, the labels of sliced samples are the same as the original time series. During test time, each slice from a test time series is classified using majority voting. For anomaly detection problem, the anomaly label will be sliced along with value series.

Window warping is a unique augmentation method for time series. Similar to dynamic time warping (DTW), this method selects a random time range, then compresses (down sample) or extends (up sample) it, while keeps other time range unchanged. Window warping would change the total length of the original time series, so it should be conducted along with window cropping for deep learning models. This method contains the normal down sampling which takes down sample through the whole length of the original time series.

Flipping is another method that generates the new sequence $x_{1}^{\prime}, \cdots, x_{N}^{\prime}$ by flipping the sign of original time series $x_{1}, \cdots, x_{N}$, where $x_{t}^{\prime}=-x_{t}$. The labels are still the same, for both anomaly detection and classification, assuming that we have symmetry between up and down directions.

Another interesting perturbation and also ensemble based method is introduced in [Fawaz et al., 2018]. This method generates new time series with DTW and then ensembles them by a weighted version of the Barycentric Averaging (DBA) algorithm. It shows improvement of classification in some of the UCR datasets.

Noise injection is a method by injecting small amount of noise/outlier into time series without changing the corresponding labels. This includes injecting Gaussian noise, spike, step-like trend, and slope-like trend, etc. For spike, we can randomly pick index and direction, randomly assign magnitude but bounded by multiples of standard deviation of the original time series. For step-like trend, it is the cumulative summation of the spikes from left index to right index. The slope-like trend is adding a linear trend into the original time series. These schemes are mostly mentioned in [Wen and Keyes, 2019]

In time series anomaly detection, the anomalies generally last long enough during a continuous span so that the start and end points are sometimes "blurry". As a result, a data point close to a labeled anomaly in terms of both time distance and value distance is very likely to be an anomaly. In this case, the label expansion method is proposed to change those data points and their labels as anomalies (by assign it an anomaly score or switch its label), which brings performance improvement for time series anomaly detection as shown in [Gao et al., 2020].

\subsection{Frequency Domain}

While most of the existing data augmentation methods focus on time domain, only a few studies investigate data augmentation from frequency domain perspective for time series.

A recent work in [Gao et al., 2020] proposes to utilize perturbations in both amplitude spectrum and phase spectrum in frequency domain for data augmentation in time series anomaly detection by convolutional neural network. Specifically, for the input time series $x_{1}, \cdots, x_{N}$, its frequency spectrum $F\left(\omega_{k}\right)$ through Fourier transform is calculated as:

$$
F\left(\omega_{k}\right)=\frac{1}{N} \sum_{t=0}^{N-1} x_{t} e^{-j \omega_{k} t}=A\left(\omega_{k}\right) \exp \left[j \theta\left(\omega_{k}\right)\right]
$$

where $\omega_{k}=\frac{2 \pi k}{N}$ is the angular frequency, $A\left(\omega_{k}\right)$ is the amplitude spectrum, and $\theta\left(\omega_{k}\right)$ is the phase spectrum. For perturbations in amplitude spectrum $A\left(\omega_{k}\right)$, the amplitude values of randomly selected segments are replaced with Gaussian noise by considering the original mean and variance in the amplitude spectrum. While for perturbations in phase spectrum $\theta\left(\omega_{k}\right)$, the phase values of randomly selected segments are added by an extra zero-mean Gaussian noise in the phase spectrum. The amplitude and phase perturbations (APP) based data augmentation combined with aforementioned time-domain augmentation methods bring significant 
time series anomaly detection improvements as shown in the experiments of [Gao et al., 2020].

Another recent work in [Lee et al., 2019] proposes to utilize the surrogate data to improve the classification performance of rehabilitative time series in deep neural network. Two conventional types of surrogate time series are adopted in the work: the amplitude adjusted Fourier transform (AAFT) and the iterated AAFT (IAAFT) [Schreiber and Schmitz, 2000]. The main idea is to perform random phase shuffle in phase spectrum after Fourier transform and then perform rank-ordering of time series after inverse Fourier transform. The generated time series from AAFT and IAAFT can approximately preserve the temporal correlation, power spectra, and the amplitude distribution of the original time series. In the experiments of [Lee et al., 2019], the authors conducted two types of data augmentation by extending the data by 10 then 100 times through AAFT and IAAFT methods, and demonstrated promising classification accuracy improvements compared to the original time series without data augmentation.

\subsection{Time-Frequency Domain}

Time-frequency analysis is a widely applied technique for time series analysis, which can be utilized as an appropriate input features in deep neural networks. However, similar to data augmentation in frequency domain, only a few studies considered data augmentation from time-frequency domain for time series.

The authors in [Steven Eyobu and Han, 2018] adopt short Fourier transform (STFT) to generate time-frequency features for sensor time series, and conduct data augmentation on the time-frequency features for human activity classification by a deep LSTM neural network. Specifically, two augmentation techniques are proposed. One is the local averaging based on a defined criteria with the generated features appended at the tail end of the feature set. Another is the shuffling of feature vectors to create variation in the data. Similarly, in speech time series, recently SpecAugment [Park et al., 2019] is proposed to make data augmentation in Mel-Frequency (a timefrequency representation based on STFT for speech time series), where the augmentation scheme consists of warping the features, masking blocks of frequency channels, and masking blocks of time steps. They demonstrate that SpecAugment can greatly improve the performance of speech recognition neural networks and obtain state-of-the-art results.

For illustration, we summarize several typical time series data augmentation methods in time, frequency, and timefrequency domains in Fig. 2.

\section{Advanced Data Augmentation Methods}

\subsection{Decomposition-based Methods}

Decomposition-based time series augmentation has also been adopted and shown success in many time series related tasks, such as forecasting and anomaly detection. Common decomposition method like STL [Cleveland et al., 1990] or RobustSTL [Wen et al., 2019b] decomposes time series $x_{t}$ as

$$
x_{t}=\tau_{t}+s_{t}+r_{t}, \quad t=1,2, \ldots N
$$
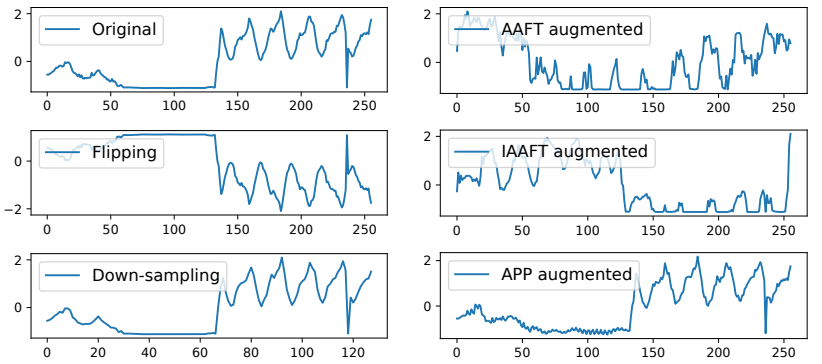

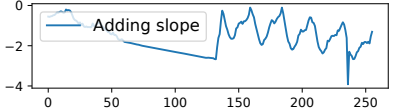

(a) time domain

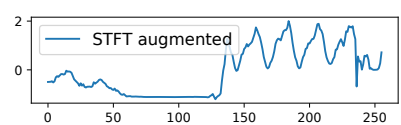

(b) (time-)frequency domain
Figure 2: Illustration of several typical time series data augmentations in time, frequency, and time-frequency domains.

where $\tau_{t}$ is the trend signal, $s_{t}$ is the seasonal/periodic signal, and the $r_{t}$ denotes the remainder signal.

In [Kegel et al., 2018], authors discussed the decomposition method to generate new time series. After STL, it recombines new time series with a deterministic component and a stochastic component. The deterministic part is reconstructed by adjusting weights for base, trend, and seasonality. The stochastic part is generated by building a composite statistical model based on residual, such as an auto-regressive model. The summed generated time series is validated by examining whether a feature-based distance to its original signal is within certain range. Meanwhile, authors in [Bergmeir et al., 2016] proposed to apply bootstrapping on the STL decomposed residuals to generate augmented signals, which are then added back with trend and seasonality to assemble a new time series. An ensemble of the forecasting models on the augmented time series has outperformed the original forecasting model consistently, demonstrating the effectiveness of decomposition-based time series augmentation approaches.

Recently, in [Gao et al., 2020], authors showed that applying time-domain and frequency-domain augmentation on the decomposed residual that is generated using robust decomposition [Wen et al., 2020; Wen et al., 2019a] can help increase the performance of anomaly detection significantly, compared with the same method without augmentation.

\subsection{Statistical Generative Models}

Time series augmentation approaches based on statistical generative models typically involve modelling the dynamics of the time series with statistical models. In [Cao et al., 2014], authors proposed a parsimonious statistical model, known as mixture of Gaussian trees, for modeling multi-modal minority class time series data to solve the problem of imbalanced classification, which shows advantages compared with existing oversampling approaches that do not exploit time series correlations between neighboring points. Authors in [Smyl and Kuber, 2016] use samples of parameters and forecast paths calculated by a statistical algorithm called LGT (Local and Global Trend). More recently, in [Kang et al., 2020] researchers use mixture autoregressive (MAR) models to simulate sets of time series and investigate the diversity and cover- 
age of the generated time series in a time series feature space.

Essentially, these models describe the conditional distribution of time series by assuming the value at time $t$ depends on previous points. Once the initial value is perturbed, a new time series sequence could be generated following the conditional distribution.

\subsection{Learning-based Methods}

Time series data augmentation methods should be capable of not only generating diverse samples, but also mimicking the characteristics of real data. In this section, we summarize some recent learning based schemes that have such potentials.

\section{Embedding Space}

In [DeVries and Taylor, 2017], the data augmentation is proposed to perform in the learned embedding space (aka., latent space). It assumes that simple transforms applied to encoded inputs rather than the raw inputs would produce more plausible synthetic data due to the manifold unfolding in feature space. Note that the selection of the representation model in this framework is open and depends on the specific task and data type. When the time series data is addressed, a sequence autoencoder is selected in [DeVries and Taylor, 2017]. Specifically, the interpolation and extrapolation are applied to generate new samples. The first $k$ nearest labels in the transformed space with the same label are identified. Then for each pair of neighboring samples, a new sample is generated which is the linear combination of them. The difference of interpolation and extrapolation lies in the weight selection in sample generation. This technique is particular useful for time series classification as demonstrated in [DeVries and Taylor, 2017]. Recently, another data augmentation method in the embedding space named MODALS (Modalityagnostic Automated Data Augmentation in the Latent Space) is proposed in [Cheung and Yeung, 2021]. Instead of training an autoencoder to learn the latent space and generate additional synthetic data for training, the MODALS method train a classification model jointly with different compositions of latent space augmentations, which demonstrates superior performance for time series classification problems.

\section{Deep Generative Models}

Deep generative models (DGMs) have recently been shown to be able to generate near-realistic high-dimensional data objects such as images and sequences. DGMs developed for sequential data, such as audio and text, often can be extended to model time series data. Among DGMs, generative adversarial networks (GANs) are popular methods to generate synthetic samples and increase the training set effectively. Although the GAN frameworks have received significant attention in many fields, how to generate effective time series data still remains a challenging problem. In this subsection, we briefly review several recent works on GANs for time series data augmentation.

In [Esteban et al., 2017], a Recurrent GAN (RGAN) and Recurrent Conditional GAN (RCGAN) are proposed to produce realistic real-valued multi-dimensional time series data. The RGAN adopts RNN in the generator and discriminator, while the RCGAN adopts both RNNs conditioned on auxiliary information. Besides desirable performance of RGAN and RCGAN for time series data augmentation, differential privacy can be used in training the RCGAN for stricter privacy guarantees like medicine or other sensitive domains. Recently, [Yoon et al., 2019] proposed TimeGAN, a natural framework for generating realistic time series data in various domains. TimeGAN is a generative time series model, trained adversarially and jointly via a learned embedding space with both supervised and unsupervised losses. Specifically, a stepwise supervised loss is introduced to learn the stepwise conditional distributions in data. It also introduces an embedding network to provide a reversible mapping between features and latent representations to reduce the high-dimensionality of the adversarial learning space. Note that the supervised loss is minimized by jointly training both the embedding and generator networks.

\section{Automated Data Augmentation}

The idea of automated data augmentation is to automatically search for optimal data augmentation policies through reinforcement learning, meta learning, or evolutionary search [Ratner et al., 2017; Cubuk et al., 2019; Zhang et al., 2020; Cheung and Yeung, 2021]. The TANDA (Transformation Adversarial Networks for Data Augmentations) scheme in [Ratner et al., 2017] is designed to train a generative sequence model over specified transformation functions using reinforcement learning in a GAN-like framework to generate realistic transformed data points, which yields strong gains over common heuristic data augmentation methods for a range of applications including image recognition and natural language understanding tasks. [Cubuk et al., 2019] proposes a procedure called AutoAugment to automatically search for improved data augmentation policies in a reinforcement learning framework. It adopts a controller RNN network to predicts an augmentation policy from the search space and another network is trained to achieve convergence accuracy. Then, the accuracy is used as reward to update the RNN controller for better policies in the next iteration. The experimental results show that AutoAugment improves the accuracy of modern image classifiers significantly in a wide range of datasets.

For time series data augmentation, the MODALS [Cheung and Yeung, 2021] is designed to find the optimal composition of latent space transformations for data augmentation using evolution search strategy based on population based augmentation (PBA) [Ho et al., 2019], which demonstrates superior performance on classification problems in continuous and discrete time series data. Another recent work on automated data augmentation is proposed in [Fons et al., 2021], where two sample-adaptive automatic weighting schemes are designed specifically for time series data: one learns to weight the contribution of the augmented samples to the loss, and the other selects a subset of transformations based on the ranking of the predicted training loss. Both adaptive policies demonstrate improvement on classification problems in multiple time series datasets.

\section{Preliminary Evaluation}

In this section, we demonstrate preliminary evaluations in three common time series tasks to show the effectiveness of data augmentation for performance improvement. 


\subsection{Time Series Classification}

In this experiment, we compare the classification performance with and without data augmentation. Specifically, we collect 5000 time series of one-week long and 5-min interval samples with binary class labels (seasonal or non-seasonal) from Alibaba Cloud monitoring system. The data is randomly splitted into training and test sets where training contains $80 \%$ of total samples. We train a fully convolutional network [Wang et al., 2017] to classify each time series in the training set. In our experiment, we inject different types of outliers, including spike, step, and slope, into the test set to evaluate the robustness of the trained classifier. The data augmentations methods applied include cropping, warping, and flipping. Table 1 summarizes the accuracies with and without data augmentation when different types of outliers are injected into the test set. It can be observed that data augmentation leads to $0.1 \% \sim 1.9 \%$ accuracy improvement.

\begin{tabular}{c|ccc}
\hline Outlier injection & w/o aug & w/ aug & Improvement \\
\hline spike & $96.26 \%$ & $96.37 \%$ & $0.11 \%$ \\
step & $93.70 \%$ & $95.62 \%$ & $1.92 \%$ \\
slope & $95.84 \%$ & $96.16 \%$ & $0.32 \%$ \\
\hline
\end{tabular}

Table 1: Accuracy improvement from data augmentation under outlier injection in time series classification.

\subsection{Time Series Anomaly Detection}

Given the challenges of both data scarcity and data imbalance in time series anomaly detection, it is beneficial by adopting data augmentation to generate more labeled data. We briefly summarize the results in [Gao et al., 2020], where a U-Net based network is designed and evaluated on public Yahoo! dataset [Laptev et al., 2015] for time series anomaly detection. The performance comparison under different settings are summarized in Table 2, including applying the model on the raw data (U-Net-Raw), on the decomposed residuals (U-Net-DeW), and on the residuals with data augmentation (U-Net-DeWA). The applied data augmentation methods include flipping, cropping, label expansion, and APP based augmentation in frequency domain. It can be observed that the decomposition helps the increase of the F1 score and the data augmentation further boosts the performance.

\begin{tabular}{c|ccc}
\hline Algorithm & Precision & Recall & F1 \\
\hline U-Net-Raw & 0.473 & 0.351 & 0.403 \\
U-Net-DeW & 0.793 & 0.569 & 0.662 \\
U-Net-DeWA (w/ aug) & 0.859 & 0.581 & 0.693 \\
\hline
\end{tabular}

Table 2: Time series anomaly detection improvement from data augmentation based on precision, recall, and F1 score.

\subsection{Time Series Forecasting}

In this subsection we demonstrate the practical effectiveness of data augmentation in two popular deep models DeepAR [Salinas et al., 2019] and Transformer [Vaswani et al., 2017]. In Table 3, we report the performance improvement on mean absolute scaled error (MASE) on several public datasets: electricity and traffic from UCI Learning Repository ${ }^{1}$ and

\footnotetext{
${ }^{1}$ http://archive.ics.uci.edu/ml/datasets.php
}

3 datasets from the M4 competition ${ }^{2}$. We consider the basic augmentation methods including cropping, warping, flipping, and APP based augmentation in frequency domain. In Table 3, we summarize average MASE without augmentation, with augmentation and average relative improvement (ARI) which is computed as the mean of (MASE $\mathrm{M}_{\mathrm{w} \text { aug }}-$

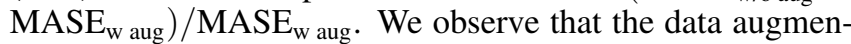
tation methods bring promising results for all models in average sense. However, the negative results can still be observed for specific data/model pairs. As a future work, it motivates us to search for advanced automated data augmentation policies that stabilize the influence of data augmentation specifically for time series forecasting.

\begin{tabular}{l|rrr|rrr}
\hline \multirow{2}{*}{ Dataset } & \multicolumn{3}{|c|}{ DeepAR } & \multicolumn{3}{c}{ Transformer } \\
\cline { 2 - 7 } & w/o aug & w/ aug & ARI & w/o aug & w/ aug & ARI \\
\hline electricity & 0.87 & 0.97 & $1.92 \%$ & 1.04 & 1.11 & $-2 \%$ \\
traffic & 0.66 & 0.80 & $-12 \%$ & 0.70 & 0.91 & $-16 \%$ \\
m4-hourly & 6.33 & 5.35 & $56 \%$ & 7.77 & 7.87 & $38 \%$ \\
m4-daily & 4.88 & 4.48 & $10 \%$ & 7.85 & 7.38 & $37 \%$ \\
m4-weekly & 12.00 & 9.34 & $76 \%$ & 6.62 & 7.09 & $23 \%$ \\
\hline
\end{tabular}

Table 3: Time seires forecasting improvement from data augmentation based on MASE.

\section{Discussion for Future Opportunities}

\subsection{Augmentation in Time-Frequency Domain}

As discussed in Section 2.3, so far there are only limited studies of time series data augmentation methods based on STFT in the time-frequency domain. Besides STFT, wavelet transform and its variants including continuous wavelet transform (CWT) and discrete wavelet transform (DWT), are another family of adaptive time-frequency domain analysis methods to characterize time-varying properties of time series. Compared to STFT, they can handle non-stationary time series and non-Gaussian noises more effectively and robustly. Among many wavelet transform variants, maximum overlap discrete wavelet transform (MODWT) is especially attractive for time series analysis [Percival and Walden, 2000; Wen et al., 2021] due to the following advantages: 1) more computationally efficiency compared to CWT; 2) ability to handle any time series length; 3) increased resolution at coarser scales compared with DWT. MODWT based surrogate time series have been proposed in [Keylock, 2006], where wavelet iterative amplitude adjusted Fourier transform (WIAAFT) is designed by combining the iterative amplitude adjusted Fourier transform (IAAFT) scheme to each level of MODWT coefficients. In contrast to IAAFT, WIAAFT does not assume sationarity and can roughly maintain the shape of the original data in terms of the temporal evolution. Besides WIAAFT, we can also consider the perturbation of both amplitude spectrum and phase spectrum as [Gao et al., 2020] at each level of MODWT coefficients as a data augmentation scheme.

It would be an interesting future direction to investigate how to exploit different wavelet transforms (CWT, DWT, MODWT, etc.) for an effective time-frequency domain based time series data augmentation in deep neural networks.

\footnotetext{
${ }^{2}$ https://github.com/Mcompetitions/M4-methods/tree/master/ Dataset
} 


\subsection{Augmentation for Imbalanced Class}

In time series classification, class imbalance occurs very frequently. One classical approach addressing imbalanced classification problem is to oversample the minority class as the synthetic minority oversampling technique (SMOTE) [Fernández et al., 2018] to artificially mitigate the imbalance. However, this oversampling strategy may change the distribution of raw data and cause overfitting. Another approach is to design cost-sensitive model by using adjust loss function [Geng and Luo, 2018]. Furthermore, [Gao et al., 2020] designed label-based weight and value-based weight in the loss function in convolution neural networks, which considers weight adjustment for class labels and the neighborhood of each sample. Thus, both class imbalance and temporal dependency are explicitly considered.

Performing data augmentation and weighting for imbalanced class together would be an interesting and effective direction. A recent study investigates this topic in the area of CV and NLP [Hu et al., 2019], which significantly improves text and image classification in low data regime and imbalanced class problems. In future, it is interesting to design deep network by jointly considering data augmentation and weighting for imbalanced class in time series data.

\subsection{Augmentation Selection and Combination}

Given different data augmentation methods summarized in Fig. 1, one key strategy is how to select and combine various augmentation methods together. The experiments in [Um et al., 2017] show that the combination of three basic timedomain methods (permutation, rotation, and time warping) is better than that of a single method and achieves the best performance in time series classification. Also, the results in [Rashid and Louis, 2019] demonstrate substantial performance improvement for a time series classification task when using a deep neural network by combining four data augmentation methods (i.e, jittering, scaling, rotation and timewarping). However, considering various data augmentation methods, directly combining different augmentations may result in a huge amount of data, and may not be efficient and effective for performance improvement. Recently, RandAugment [Cubuk et al., 2020] is proposed as a practical way for augmentation combination in image classification and object detection. For each random generated dataset, RandAugment is based on only two interpretable hyperparameters $N$ (number of augmentation methods to combine) and $M$ (magnitude for all augmentation methods), where each augmentation is randomly selected from $K=14$ available augmentation methods. Furthermore, this randomly combined augmentation with simple grid search can be used in the reinforcement learning based data augmentation as [Cubuk et al., 2019] for efficient space searching.

An interesting future direction is how to design effective augmentation selection and/or combination strategies suitable for time series data in deep learning. Customized reinforcement learning and meta learning optimized for time series could be potential approaches. Furthermore, algorithm efficiency is another important consideration in practice.

\subsection{Augmentation with Gaussian Processes}

Gaussian Processes (GPs) [Rasmussen and Williams, 2005] are well-known Bayesian non-parametric models suitable for time series analysis [Roberts et al., 2013]. From the functionspace view, GPs induce a distribution over functions, i.e., a stochastic process. Time series can be viewed as functions with time as input and observation as output, and thus can be modeled with GPs. A GP $f(t) \sim \mathcal{G} \mathcal{P}\left(m(t), k\left(t, t^{\prime}\right)\right)$ is characterized by a mean function $m(t)$ and a covariance kernel function $k\left(t, t^{\prime}\right)$. The choice of the kernel allows to place assumptions on some general properties of the modeled functions, such as smoothness, scale, periodicity and noise level. Kernels can be composed through addition and multiplication, resulting in compositional function properties, such as pseudo-periodicity, additive decomposability, and change point. GPs are often applied to interpolation and extrapolation tasks, which correspond to imputation and forecasting in time series analysis. Furthermore, deep Gaussian processes(DGPs) [Damianou and Lawrence, 2013; Salimbeni and Deisenroth, 2017], which are richer models with hierarchical composition of GPs and often exceed standard (single-layer) GPs significantly in many cases, have not been well studied for time series. We believe GPs and DGPs are future directions as they allow to sample time series with those properties mentioned above through the design of kernels, and to generate new data instances from existing ones by exploiting their interpolation/extrapolation abilities.

\subsection{Augmentation with Deep Generative Models}

Current DGMs adopted for time series data augmentation are mainly GANs. However, other DGMs also have great potentials for time series modeling. For example, deep autoregressive networks (DARNs) exhibit a natural fit for time series because they generate data in a sequential manner, obeying the causal direction of physical time series data generating process. DARNs like Wavenet [Oord et al., 2016] and Transformer [Vaswani et al., 2017] have demonstrated promising performance in time series forecasting tasks [Alexandrov et al., 2020]. Another example is normalizing flows (NFs) [Kobyzev et al., 2020], which recently have shown success in modeling time series stochastic processes with excellent inter-/extrapolation performance given observed data [Deng et al., 2020]. Most recently, variational autoencoders (VAEs) based data augmentation [Fu et al., 2020] are investigated for human activity recognition.

In summary, besides the common GAN architectures, how to leverage other deep generative models like DARNs, NFs, and VAEs, which are less investigated for time series data augmentation, remain exciting future opportunities.

\section{Conclusion}

As deep learning models are becoming more popular on time series data, the limited labeled data calls for effective data augmentation methods. In this paper, we give a comprehensive survey on time series data augmentation methods in various tasks. We organize the reviewed methods in a taxonomy consisting of basic and advanced approaches, summarize representative methods in each category, compare them empirically in typical tasks, and highlight future research directions. 


\section{References}

[Alexandrov et al., 2020] Alexander Alexandrov, Konstantinos Benidis, Michael Bohlke-Schneider, Valentin Flunkert, Jan Gasthaus, Tim Januschowski, Danielle C Maddix, Syama Rangapuram, David Salinas, Jasper Schulz, et al. Gluonts: Probabilistic and neural time series modeling in python. Journal of Machine Learning Research, 21(116):1-6, 2020.

[Bandara et al., 2020] Kasun Bandara, Hansika Hewamalage, Yuan-Hao Liu, Yanfei Kang, and Christoph Bergmeir. Improving the accuracy of global forecasting models using time series data augmentation. arXiv preprint arXiv:2008.02663, 2020.

[Bergmeir et al., 2016] Christoph Bergmeir, Rob J. Hyndman, and José M. Benítez. Bagging exponential smoothing methods using STL decomposition and Box-Cox transformation. International Journal of Forecasting, 32(2):303-312, 2016.

[Cao et al., 2014] Hong Cao, Vincent YF Tan, and John ZF Pang. A parsimonious mixture of gaussian trees model for oversampling in imbalanced and multimodal time-series classification. IEEE TNNLS, 25(12):2226-2239, 2014.

[Cheung and Yeung, 2021] Tsz-Him Cheung and Dit-Yan Yeung. MODALS: Modality-agnostic automated data augmentation in the latent space. In International Conference on Learning Representations (ICLR), 2021.

[Cleveland et al., 1990] Robert B Cleveland, William S Cleveland, Jean E McRae, and Irma Terpenning. STL: A seasonal-trend decomposition procedure based on loess. Journal of Official Statistics, 6(1):3-73, 1990.

[Cubuk et al., 2019] Ekin D. Cubuk, Barret Zoph, Dandelion Mane, Vijay Vasudevan, and Quoc V. Le. AutoAugment: Learning augmentation strategies from data. In IEEE CVPR 2019, pages 113123, June 2019.

[Cubuk et al., 2020] Ekin D Cubuk, Barret Zoph, Jonathon Shlens, and Quoc V Le. RandAugment: Practical automated data augmentation with a reduced search space. In 2020 IEEE/CVF Conference on Computer Vision and Pattern Recognition Workshops, pages 3008-3017, 2020.

[Cui et al., 2015] X Cui, V Goel, and B Kingsbury. Data augmentation for deep neural network acoustic modeling. IEEE/ACM TASLP, 23(9):1469-1477, 2015.

[Cui et al., 2016] Zhicheng Cui, Wenlin Chen, et al. Multi-scale convolutional neural networks for time series classification. arXiv preprint arXiv:1603.06995, 2016.

[Damianou and Lawrence, 2013] Andreas Damianou and Neil D Lawrence. Deep gaussian processes. In Artificial intelligence and statistics, pages 207-215. PMLR, 2013.

[Deng et al., 2020] Ruizhi Deng, Bo Chang, Marcus A Brubaker, Greg Mori, and Andreas Lehrmann. Modeling continuous stochastic processes with dynamic normalizing flows. In NeurIPS 2020, Dec 2020.

[DeVries and Taylor, 2017] Terrance DeVries and Graham W. Taylor. Dataset augmentation in feature space. In ICLR 2017, pages 1-12, Toulon, 2017.

[Esteban et al., 2017] Cristóbal Esteban, Stephanie L Hyland, and Gunnar Rätsch. Real-valued (medical) time series generation with recurrent conditional gans. arXiv preprint arXiv:1706.02633, 2017.

[Fawaz et al., 2018] Hassan Ismail Fawaz, Germain Forestier, Jonathan Weber, Lhassane Idoumghar, and Pierre-Alain Muller.
Data augmentation using synthetic data for time series classification with deep residual networks. In ECML/PKDD Workshop on AALTD, 2018.

[Fawaz et al., 2019] Hassan Ismail Fawaz, Germain Forestier, Jonathan Weber, et al. Deep learning for time series classification: a review. Data Mining and Knowledge Discovery, 33(4):917-963, 2019.

[Fernández et al., 2018] Alberto Fernández, Salvador Garcia, Francisco Herrera, and Nitesh V Chawla. SMOTE for learning from imbalanced data: progress and challenges, marking the 15-year anniversary. Journal of Artificial Intelligence Research, 61:863905, 2018.

[Fons et al., 2021] Elizabeth Fons, Paula Dawson, Xiao-jun Zeng, John Keane, and Alexandros Iosifidis. Adaptive weighting scheme for automatic time-series data augmentation. arXiv preprint arXiv:2102.08310, 2021.

[Fu et al., 2020] Biying Fu, Florian Kirchbuchner, and Arjan Kuijper. Data augmentation for time series: traditional vs generative models on capacitive proximity time series. In ACM PETRA, pages 1-10, 2020.

[Gamboa, 2017] John Cristian Borges Gamboa. Deep learning for time-series analysis. arXiv preprint arXiv:1701.01887, 2017.

[Gao et al., 2020] Jingkun Gao, Xiaomin Song, Qingsong Wen, Pichao Wang, Liang Sun, and Huan Xu. Robusttad: Robust time series anomaly detection via decomposition and convolutional neural networks. MileTS'20: 6th KDD Workshop on Mining and Learning from Time Series, pages 1-6, 2020.

[Geng and Luo, 2018] Yue Geng and Xinyu Luo. Cost-sensitive convolution based neural networks for imbalanced time-series classification. arXiv preprint arXiv:1801.04396, 2018.

[Han et al., 2019] Z Han, J Zhao, H Leung, K F Ma, and W Wang. A review of deep learning models for time series prediction. IEEE Sensors Journal, page 1, 2019.

[Ho et al., 2019] Daniel Ho, Eric Liang, Xi Chen, Ion Stoica, and Pieter Abbeel. Population based augmentation: Efficient learning of augmentation policy schedules. In International Conference on Machine Learning (ICML), pages 2731-2741, 2019.

[Hu et al., 2019] Zhiting Hu, Bowen Tan, Russ R Salakhutdinov, Tom M Mitchell, and Eric P Xing. Learning data manipulation for augmentation and weighting. In NeurIPS 2019, pages 15764 15775, 2019.

[Hu et al., 2020] Hailin Hu, MingJian Tang, and Chengcheng Bai. Datsing: Data augmented time series forecasting with adversarial domain adaptation. In Proceedings of the 29th ACM International Conference on Information \& Knowledge Management, pages 2061-2064, 2020.

[Iwana and Uchida, 2020] Brian Kenji Iwana and Seiichi Uchida. An empirical survey of data augmentation for time series classification with neural networks. arXiv preprint arXiv:2007.15951, 2020.

[Kang et al., 2020] Yanfei Kang, Rob J Hyndman, and Feng Li. GRATIS: Generating time series with diverse and controllable characteristics. Statistical Analysis and Data Mining: The ASA Data Science Journal, 13(4):354-376, 2020.

[Kegel et al., 2018] Lars Kegel, Martin Hahmann, and Wolfgang Lehner. Feature-based comparison and generation of time series. In SSDBM 2018, 2018.

[Keylock, 2006] C J Keylock. Constrained surrogate time series with preservation of the mean and variance structure. Phys. Rev. E, 73(3):36707, Mar 2006. 
[Kobyzev et al., 2020] Ivan Kobyzev, Simon Prince, and Marcus Brubaker. Normalizing flows: An introduction and review of current methods. IEEE Transactions on Pattern Analysis and Machine Intelligence, pages 1-17, 2020.

[Krizhevsky et al., 2012] Alex Krizhevsky, Ilya Sutskever, and Geoffrey E Hinton. Imagenet classification with deep convolutional neural networks. In NeurIPS 2012, pages 1097-1105, 2012.

[Laptev et al., 2015] Nikolay Laptev, Saeed Amizadeh, et al. Generic and scalable framework for automated time-series anomaly detection. KDD, pages 1939-1947, 2015.

[Le Guennec et al., 2016] Arthur Le Guennec, Simon Malinowski, and Romain Tavenard. Data augmentation for time series classification using convolutional neural networks. In ECML/PKDD Workshop on AALTD, 2016.

[Lee and Kim, 2020] Si Woon Lee and Ha Young Kim. Stock market forecasting with super-high dimensional time-series data using convlstm, trend sampling, and specialized data augmentation. Expert Systems with Applications, 161:113704, 2020.

[Lee et al., 2019] Tracey Kah-Mein Lee, YL Kuah, Kee-Hao Leo, Saeid Sanei, Effie Chew, and Ling Zhao. Surrogate rehabilitative time series data for image-based deep learning. In EUSIPCO 2019, pages 1-5, 2019.

[Lim et al., 2018] Swee Kiat Lim, Yi Loo, Ngoc-Trung Tran, NgaiMan Cheung, Gemma Roig, and Yuval Elovici. DOPING: Generative data augmentation for unsupervised anomaly detection with gan. In 2018 IEEE International Conference on Data Mining (ICDM), pages 1122-1127. IEEE, 2018.

[Oord et al., 2016] Aaron van den Oord, Sander Dieleman, Heiga Zen, Karen Simonyan, Oriol Vinyals, Alex Graves, Nal Kalchbrenner, Andrew Senior, and Koray Kavukcuoglu. Wavenet: A generative model for raw audio. In International Conference on Learning Representations, 2016.

[Park et al., 2019] Daniel S Park, William Chan, Yu Zhang, ChungCheng Chiu, Barret Zoph, Ekin D Cubuk, et al. SpecAugment: A simple data augmentation method for automatic speech recognition. In INTERSPEECH 2019, pages 2613-2617, 2019.

[Percival and Walden, 2000] Donald B Percival and Andrew T Walden. Wavelet methods for time series analysis, volume 4. Cambridge university press, New York, 2000.

[Rashid and Louis, 2019] Khandakar M Rashid and Joseph Louis. Times-series data augmentation and deep learning for construction equipment activity recognition. Advanced Engineering Informatics, 42:100944, 2019.

[Rasmussen and Williams, 2005] Carl Edward Rasmussen and Christopher KI Williams. Gaussian Processes for Machine Learning. The MIT Press, 2005.

[Ratner et al., 2017] Alexander J Ratner, Henry R Ehrenberg, Zeshan Hussain, Jared Dunnmon, and Christopher Ré. Learning to compose domain-specific transformations for data augmentation. NeurIPS 2017, 30:3239, 2017.

[Roberts et al., 2013] Stephen Roberts, Michael Osborne, Mark Ebden, Steven Reece, Neale Gibson, and Suzanne Aigrain. Gaussian processes for time-series modelling. Philosophical Transactions of the Royal Society A: Mathematical, Physical and Engineering Sciences, 371(1984):20110550, 2013.

[Salimbeni and Deisenroth, 2017] Hugh Salimbeni and Marc Peter Deisenroth. Doubly stochastic variational inference for deep gaussian processes. In NeurIPS 2017, pages 4591-4602, 2017.
[Salinas et al., 2019] David Salinas, Valentin Flunkert, Jan Gasthaus, and Tim Januschowski. DeepAR: Probabilistic forecasting with autoregressive recurrent networks. International Journal of Forecasting, 2019.

[Schreiber and Schmitz, 2000] Thomas Schreiber and Andreas Schmitz. Surrogate time series. Physica D: Nonlinear Phenomena, 142(3):346-382, 2000.

[Shorten and Khoshgoftaar, 2019] Connor Shorten and Taghi M Khoshgoftaar. A survey on image data augmentation for deep learning. Journal of Big Data, 6(1):60, 2019.

[Smyl and Kuber, 2016] Slawek Smyl and Karthik Kuber. Data preprocessing and augmentation for multiple short time series forecasting with recurrent neural networks. In 36th International Symposium on Forecasting, June 2016.

[Steven Eyobu and Han, 2018] Odongo Steven Eyobu and Dong Seog Han. Feature representation and data augmentation for human activity classification based on wearable IMU sensor data using a deep LSTM neural network. Sensors, 18(9):2892, 2018.

[Um et al., 2017] Terry T Um, Franz M J Pfister, Daniel Pichler, Satoshi Endo, Muriel Lang, Sandra Hirche, Urban Fietzek, and Dana Kulić. Data augmentation of wearable sensor data for Parkinson's disease monitoring using convolutional neural networks. In ACM ICMI 2017, pages 216-220, 2017.

[Vaswani et al., 2017] Ashish Vaswani, Noam Shazeer, Niki Parmar, Jakob Uszkoreit, Llion Jones, et al. Attention is all you need. In NeurIPS 2017, pages 5998-6008, 2017.

[Wang et al., 2017] Zhiguang Wang, Weizhong Yan, and Tim Oates. Time series classification from scratch with deep neural networks: A strong baseline. In IJCNN, pages 1578-1585, 2017.

[Wen and Keyes, 2019] Tailai Wen and Roy Keyes. Time series anomaly detection using convolutional neural networks and transfer learning. In IJCAI Workshop on AI4IoT, 2019.

[Wen et al., 2019a] Qingsong Wen, Jingkun Gao, Xiaomin Song, Liang Sun, and Jian Tan. RobustTrend: A Huber loss with a combined first and second order difference regularization for time series trend filtering. In IJCAI, pages 3856-3862, 2019.

[Wen et al., 2019b] Qingsong Wen, Jingkun Gao, Xiaomin Song, Liang Sun, Huan Xu, and Shenghuo Zhu. RobustSTL: A robust seasonal-trend decomposition algorithm for long time series. In AAAI, volume 33, pages 5409-5416, 2019.

[Wen et al., 2020] Qingsong Wen, Zhe Zhang, Yan Li, and Liang Sun. Fast RobustSTL: Efficient and robust seasonal-trend decomposition for time series with complex patterns. In $K D D$, pages 2203-2213, 2020.

[Wen et al., 2021] Qingsong Wen, Kai He, Liang Sun, Yingying Zhang, Min Ke, and Huan Xu. RobustPeriod: Time-frequency mining for robust multiple periodicities detection. In International Conference on Management of Data (SIGMOD), 2021.

[Yoon et al., 2019] Jinsung Yoon, Daniel Jarrett, and Mihaela van der Schaar. Time-series generative adversarial networks. In NeurIPS 2019, pages 5508-5518, 2019.

[Zhang et al., 2020] Xinyu Zhang, Qiang Wang, Jian Zhang, and Zhao Zhong. Adversarial autoaugment. In International Conference on Learning Representations (ICLR), 2020.

[Zhou et al., 2019] Bin Zhou, Shenghua Liu, Bryan Hooi, Xueqi Cheng, and Jing Ye. Beatgan: Anomalous rhythm detection using adversarially generated time series. In IJCAI, pages 4433-4439, 2019. 